\title{
RANKS OF MATRICES OVER ORE DOMAINS
}

\author{
H. BEDOYA AND J. LEWIN ${ }^{1,2}$
}

\begin{abstract}
Let $R$ be a Noetherian Ore domain. Then rank $M=$ inner rank $M$ for every matrix $M$ over $R$ if and only if $R$ is projective-free of global dimension at most 2.
\end{abstract}

1. Let $R$ be a right and left Ore domain with field of quotients $Q$ and let $M$ be a finitely generated right $R$-module. The then $\operatorname{rank} r(M)$ is the $Q$ dimension of the vector space $M \otimes_{R} Q$ and we denote by $d(M)$ the least number of elements in a set of generators of $M$.

If $\gamma$ is a homomorphism of free $R$-modules $\gamma: R^{n} \rightarrow R^{m}$, then the rank $r(\gamma)$ of $\gamma$ is the rank of the image of $\gamma$. The inner rank $\rho(\gamma)$ of $\gamma$ (defined by Bergman [1, p. 126] for arbitrary rings) may be defined to be the minimum of $d(M)$, where $\operatorname{Im}(\gamma) \leqslant M \leqslant R^{m}$. Alternatively, if $G$ is a matrix for $\gamma$, then $\rho(\gamma)$ is the least integer $\rho$ such that $G=G_{1} G_{2}$ with $G_{1}$ an $m \times \rho$ and $G_{2}$ a $\rho \times n$ matrix. Inner rank and rank do not always coincide, even over commutative domains. In this note we give necessary and sufficient conditions on a Noetherian Ore domain for the two notions of rank to coincide, and thus give a partial answer to a question raised by Bergman [1, p. 150].

2. Throughout, $R$ is a right and left Ore domain with field of quotients $Q$. All modules are right $R$-modules, and tensor products are over $R$.

Lemma 1. (a) If $0 \rightarrow N \rightarrow R^{n}$ is exact then $N \otimes Q=0$ implies that $N=0$.

(b) Let $0 \rightarrow R^{n} \rightarrow M$ be an exact sequence of $R$-modules. If $d(M) \leqslant n$ then in fact $M \cong R^{n}$.

Proof. Both parts of the lemma are immediate consequences of the exactness of $\otimes_{R} Q$.

(a) If $x$ is a nonzero element of $N$ then $x R \cong R$. Thus the exactness of $0 \rightarrow R \rightarrow N$ gives $0 \rightarrow Q \rightarrow N \otimes Q$ which insures that $N \otimes Q \neq 0$.

(b) Let $0 \rightarrow K \rightarrow R^{n} \rightarrow M \rightarrow 0$ be a presentation for $M$. Tensoring both sequences with $Q$, we get the exact diagram

Received by the editors July 12, 1974 and, in revised form, April 18, 1975.

AMS (MOS) subject classifications (1970). Primary 13A02.

Key words and phrases. Rank of matrices, Ore domain.

1 The second author gratefully acknowledges the support of the National Science Foundation.

2 The authors are pleased to thank David Lissner for some illuminating discussions. 


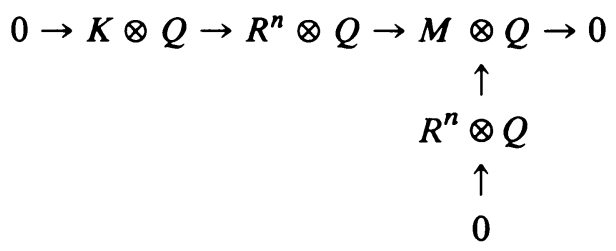

This shows that both maps into $M \otimes Q$ are isomorphisms and hence that $K \otimes Q=0$. Thus $K=0$, as needed.

LEMMA 2. The following are equivalent:

(i) If $M \leqslant R^{n}$, then $r(M)=n$ or $M \leqslant K \leqslant R^{n}$ with $K \simeq R^{n-1}$.

(ii) If $M \leqslant R^{n}$, then $r(M)=n$ or $M \leqslant K \leqslant R^{n}$ with $d(K)=n-1$.

(iii) If $0 \rightarrow K \rightarrow R^{n} \rightarrow R$ is exact, then $K \simeq R^{n-1}$.

Proof. (i) $\Rightarrow$ (ii) trivially.

Assume (ii) and let $K^{\prime}$ be the kernel of a functional $R^{n} \rightarrow R$. Tensoring with $Q$, we see $r\left(K^{\prime}\right)=n-1$. Thus if $K^{\prime} \neq R^{n-1}$, then, by Lemma $1, d\left(K^{\prime}\right) \geqslant n$. Then by (ii) $K^{\prime} \leqslant K \leqslant R^{n}$ with $d(K)=n-1$. But $K / K^{\prime}$, as a nonzero submodule of $R$, contains a copy of $R$ generated, say, by $k+K^{\prime}$. But then $k R \cap K^{\prime}=0$ so that $K^{\prime} \oplus k R \leqslant K$ and $r(K) \geqslant n$. This contradicts $d(K)$ $=n-1$ and so (iii) holds.

Assume (iii) and suppose $M \leqslant R^{n}$, with $r(M)<n$. Then there is a $Q$ functional $\gamma: R^{n} \otimes Q \rightarrow Q$ which vanishes at $M \otimes Q$. Let $\gamma^{\prime}$ be the restriction of $\gamma$ to $R^{n}$. Then $\gamma^{\prime}: R^{n} \rightarrow Q$ is an $R$-linear map which vanishes at $M$. Now $\gamma^{\prime}\left(R^{n}\right)$ is a finitely generated $R$-module, say $\gamma^{\prime}\left(R^{n}\right)=q_{1} R+\cdots+q_{n} R$. Since $R$ is also a left Ore domain, there are elements $r, r_{1}, \ldots, r_{n}$ in $R$ with $r \neq 0$ and $q_{i}=r^{-1} r_{i}$. Thus $r \gamma^{\prime}\left(R^{n}\right) \subseteq R$. Thus $r \gamma^{\prime}$ is an $R$-functional from $R^{n}$ to $R$. Since $\gamma^{\prime}(M)=0$, also $r \gamma^{\prime}(M)=0$. Thus $M \leqslant \operatorname{Ker}\left(r \gamma^{\prime}\right)$, which is isomorphic to $R^{n-1}$ by assumption, and (i) holds.

Some DEFinitions. $\gamma: R^{n} \rightarrow R^{n}$ is full if $\rho(\gamma)=n$. $R$ has $A C C^{*}$ if for each $n$, free $R$-modules have ACC on $n$-generator submodules.

Proposition. (a) Let $R$ satisfy (i) and $\gamma: R^{n} \rightarrow R^{m}$. Then $\rho(\gamma)=r(\gamma)$.

(b) Let $R$ have $A C C^{*}$. If $R$ does not satisfy (iii) then there is a full homomorphism $\gamma: R^{n} \rightarrow R^{n}$ of rank less than $n$.

Proof. (a) Assume (i) and let $\gamma: R^{n} \rightarrow R^{m}$. If $m=1$ then clearly $\rho(\gamma)$ $=r(\gamma)$ and we use induction on $m$. If $\rho(\gamma)=m$, then $\operatorname{Im} \gamma$ is not contained in an $m-1$ generator submodule of $R^{m}$. Thus, by (i), $r(\operatorname{Im} \gamma)=m$ and hence $\rho(\gamma)=r(\gamma)=m$. Otherwise $\operatorname{Im} \gamma \leqslant M \leqslant R^{m}$ with $d(M)=\rho(\gamma)<m$. Thus, by (i), $M \leqslant R^{m-1} \leqslant R^{m}$. Let $\gamma_{1}$ be the map $\gamma$ cut down to $R^{m-1}$ and $\gamma_{2}$ be the injection $R^{m-1} \rightarrow R^{m}$. Then $\gamma=\gamma_{1} \gamma_{2}$. Clearly $\rho\left(\gamma_{1}\right) \geqslant \rho(\gamma)$. But since Im $\gamma_{1} \leqslant M \leqslant R^{m-1}$ and $d(M)=\rho(\gamma)$, then $\rho\left(\gamma_{1}\right)=\rho(\gamma)$. Thus $\rho\left(\gamma_{1}\right)=r\left(\gamma_{1}\right)$ by induction. Since $\gamma_{2}$ is one-to-one, $r\left(\gamma_{1}\right)=r(\gamma)$. Thus, finally, $\rho(\gamma)=\rho\left(\gamma_{1}\right)$ $=r\left(\gamma_{1}\right)=r(\gamma)$.

(b) Assume $R$ has ACC* and that $\gamma: R^{n} \rightarrow R$ is a functional whose kernel $K$ is not isomorphic to $R^{n-1}$. Since $r(K)=n-1$, it follows from Lemma 1 that $d(K) \geqslant n$ and also that a free submodule $F$ of $K$ has $r(F)=d(F)$ 
$\leqslant n-1$. Thus $K$ is not free and, by $\mathrm{ACC}^{*}, K$ has the maximal condition on free submodules. Let then $F \leqslant K$ be a submodule of $K$ maximal with respect to being free. Then $r(F)=n-1$. Let $x$ be $K$ but not in $F$. Let $M=F+x R$. If $d(M)<n$, then $M$ is free, in contradiction with the choice of $F$. Thus $d(M)=n$. Suppose $M \leqslant T \leqslant R^{n}$ with $d(T)<n$. Then, since $F \leqslant T$, Lemma 1 insures that $T=R^{n-1}$. Now from the exact sequence $0 \rightarrow M \rightarrow T$ $\rightarrow T / M \rightarrow 0$ we get the exact sequence

$$
0 \rightarrow M \otimes Q \rightarrow T \otimes Q \rightarrow T / M \otimes Q \rightarrow 0
$$

which gives $T / M \otimes Q=0$. Now since $M \leqslant K$ we also have a sequence $T / M \rightarrow T+K / K \rightarrow 0$ which gives

$$
T / M \otimes Q \rightarrow T+K / K \otimes Q \rightarrow 0
$$

Thus $T+K / K \otimes Q$ is zero and hence by Lemma $1, T+K / K=0$, i.e. $T \leqslant K$. This contradicts the maximality of $F$. It follows that any map $\alpha: R^{n} \rightarrow R^{n}$ whose image is $M$ has inner rank $n$ and rank $n-1$.

The Proposition shows that for Ore domains with ACC, whether rank = inner rank can be decided by considering only full homomorphisms.

If $R$ is a Noetherian (and hence Ore) domain, we can couch the Proposition in homological terms.

THEOREM 1. Let $R$ be a Noetherian domain. Then inner rank = rank if and only if $R$ has global dimension at most two and finitely generated projective $R$ modules are free.

Proof. By Theorem 21 of [3], $\mathrm{gl} \operatorname{dim}(R)=1+\operatorname{hom} \operatorname{dim}(A)$ where $A$ is some ideal of $R$. Present $A$ as $0 \rightarrow K \rightarrow F \rightarrow A \rightarrow 0$ where $F$ is a finitely generated free module. If inner rank $=$ rank then (iii) holds so that hom $\operatorname{dim} A \leqslant 1$ and hence $\mathrm{gl} \operatorname{dim}(R) \leqslant 2$. Further, if $P$ is a finitely generated projective with $d=d(P)$ then $P \oplus Q=R^{d}$ for some $Q$. If $r(P)=d$ then $P$ is free. If $r(P)<d$, it follows from (i) that $P \leqslant M<R^{d}$ with $d(M)$ $=d-1$. But $P$ is again a summand of $M$, so $d(P) \leqslant d-1$, a contradiction. So finitely generated projective $R$-modules are free. The reverse implication follows in a similar manner.

The Noetherian, or at least the ACC*, hypothesis of Theorem 1 is necessary: considering matrices, let $M$ be an $m \times n$ matrix over $R$ of inner rank $\rho$. Then $M=M_{1} M_{2}$ where $M_{1}$ is $m \times \rho$ and $M_{2}$ is $\rho \times n$. Then $M$ has inner rank $\rho$ when considered as a matrix over the ring $R^{\prime}$ generated by the entries of $M_{1}$ and $M_{2}$. Also, if $R$ is commutative, $r(M)$ is the rank of $M$ as a matrix over $R^{\prime}$, since $r(M)$ is the maximal order of a submatrix of $M$ with nonzero determinant. So if $R$ is commutative and inner rank = rank for all finitely generated subrings of $R$, this is also true for $R$. Thus a union of (finitely generated) projective-free commutative rings of global dimension $\leqslant 2$ has the property that inner rank = rank. Such a ring may well have global dimension $>2$. For example let $G$ be a torsion-free infinitely generated locally cyclic abelian group. Then $\mathbf{Z} G$ has global dimension 3 [2, Theorem 5, p. 149]. 
3. Remarks. (a) David Lissner proved for us that the following is an explicit example of a full $3 \times 3$ matrix which has rank 2 : let $k$ be a field,

$$
R=k[x, y, z] \text {, and } A=\left(\begin{array}{ccc}
-z & 0 & x \\
y & -x & 0 \\
0 & z & -y
\end{array}\right)
$$

(b) It is easy to see that if every full matrix over $R[x]$ is invertible over $Q(x)$ then every full matrix over $R$ is invertible over $Q$. Theorem 1 gives a simple proof of the well-known fact that if $R$ is a Dedekind domain and $R[x]$ is projective-free then $R$ is a PID.

(c) Using results of Lissner and Geramita [4, Theorems 2.6 and 3.4], Theorem 1 can be restated in terms of the outer product property: for a commutative Noetherian domain, inner rank = rank if and only if $R$ is an outer product domain which is a UFD.

\section{REFERENCES}

1. G. Bergman, Commuting elements in free algebras and related topics in ring theory, Thesis, Harvard University, 1967.

2. K. W. Gruenberg, Cohomological topics in group theory, Lecture Notes in Math., vol. 143, Springer-Verlag, Berlin and New York, 1970. MR 43 \# 4923.

3. I. Kaplansky, Fields and rings, 2nd ed., Chicago Notes in Math., Univ. of Chicago Press, Chicago, Ill., 1972.

4. D. Lissner and A. Geramita, Remarks on OP and Towber rings, Canad. J. Math. 22 (1970), 1109-1117. MR 42 \#5972.

Department of Mathematics, Syracuse University, Syracuse, New York 13210 